\title{
Sleep Disturbance and Serum Ferritin Levels Associate with High Impulsivity and Impulse Control Disorders in Male Parkinson's Disease Patients
}

\author{
Ryan Scott Anderton 1, 2,3, *, Michelle Byrnes ${ }^{2,3}$, Alexa Jefferson ${ }^{2}$, Sue Walters ${ }^{2,3}$, Soumya Ghosh ${ }^{2,3}$, \\ Rick Stell $^{2,3}$, Frank Louis Mastaglia ${ }^{2,3,4}$ \\ ${ }^{1}$ School of Health Sciences and Institute for Health Research, University of Notre Dame Australia, Fremantle, Australia \\ ${ }^{2}$ Perron Institute for Neurological and Translational Science, Perth, Australia \\ ${ }^{3}$ Centre for Neuromuscular and Neurological Disorders, University of Western Australia, Perth, Australia \\ ${ }^{4}$ Institute for Immunology and Infectious Diseases, Murdoch University, Perth, Australia
}

Email address:

ryan.anderton@nd.edu.au (R. S. Anderton)

${ }^{*}$ Corresponding author

\section{To cite this article:}

Ryan Scott Anderton, Michelle Byrnes, Alexa Jefferson, Sue Walters, Soumya Ghosh, Rick Stell, Frank Louis Mastaglia. Sleep Disturbance and Serum Ferritin Levels Associate with High Impulsivity and Impulse Control Disorders in Male Parkinson's Disease Patients. American Journal of Psychiatry and Neuroscience. Vol. 5, No. 4, 2017, pp. 45-52. doi: 10.11648.j.ajpn.20170504.12

Received: June 26, 2017; Accepted: July 19, 2017; Published: August 26, 2017

\begin{abstract}
Impulse control disorders (ICDs) occur in a subset of Parkinson's disease (PD) patients on dopaminergic medications however there are currently no reliable markers to identify patients at risk. Sleep disturbances are more common in patients with an ICD. Serum ferritin levels have been associated with PD disease stage and progression, but have not previously been associated with impulsivity levels. The objective of this study was to determine if serum ferritin levels and sleep disturbance are associated with high traits of impulsivity and ICD in a cohort of PD patients attending a movement disorders clinic. This study assessed impulsiveness in 87 PD patients using the Barratt Impulsiveness Scale. Severity of sleep disturbance was determined using the sleep-related items of the MDS-UPDRS. Serum ferritin, iron and transferrin levels were measured in patients, as well as 36 age-matched healthy controls. Serum ferritin levels were significantly elevated in male PD patients in the high impulsivity group compared to patients in the low $(p=.022)$ and normal range groups $(p=.024)$ and showed a linear increase across the three groups. Sleep disturbance also demonstrated a linear trend, which was most severe in the high impulsivity group $(p=.030)$. A subgroup of 11 male PD patients who fulfilled the DSM-5 criteria for an ICD had significantly higher ferritin levels and more severe sleep disturbance when compared with the remaining male PD cohort. Serum ferritin levels and sleep disturbance severity are highlighted as potential markers for abnormal impulsivity and ICD in PD patients.
\end{abstract}

Keywords: Impulsivity, BIS-11, Ferritin, Sleep Disturbance, PD

\section{Introduction}

Impulse control disorders (ICDs) are becoming increasingly identified as a consequence of dopaminergic medication in Parkinson's disease (PD) [1]. Recent studies suggest ICDs occur in upwards of $40 \%$ of PD patients [2], with males more likely to report multiple impulsive behaviours than females [3]. While there are currently no reliable predictive markers for the development of an ICD, patients with higher traits of impulsivity may be more susceptible, although this is yet to be established. Patients displaying high impulsivity traits are known to have a younger age at PD onset [3] and are also more likely to be individuals who have recurrent falls [4].

Sleep deprivation and disturbances are known to increase impulsive behaviours [5]. Moreover, patients with PD experience greater sleep-related problems [6], a feature possibly exacerbated by medications such as dopamine 
agonists [7, 8]. Sleep disturbances, including sleep fragmentation and daytime sleepiness, are more common in PD patients with ICDs than PD alone and healthy controls [9]. Similar patterns of sleep disturbance have been linked to impulsive behaviour in other related disorders such as Attention Deficit/Hyperactivity Disorder (ADHD) and restless legs syndrome (RLS) [10,11], both of which have been associated with low serum levels of the iron storage protein, ferritin [12, 13]. Moreover, in a study of children with ADHD, Oner and colleagues (2012) identified a relationship between serum ferritin and hyperactivity scores [14], presenting a possible marker for broader impulse control conditions.

A number of studies have shown that there is a selective accumulation of iron in the substantia nigra in PD [15] and changes in levels of the major iron-binding protein ferritin, however it is unclear what role they play in the pathogenesis of the disease [16]. Studies examining serum levels of ferritin in PD patients have been inconclusive, showing either increased levels [17], or no significant relationship [18, 19]. Ferritin levels have been reported to be increased in both male and female PD patients when compared to controls, correlating with Hoehn \& Yahr stage and disease duration [20]. However, limited studies have investigated ferritin levels during disease progression, most importantly in the context of non-motor aspects of the disease, and an association between ferritin and impulsivity levels has not previously been investigated.

Thus, the present study investigated whether trait impulsivity was associated with sleep disturbance and serum levels of ferritin, iron and transferrin in a cohort of PD patients without a prior history of an ICD.

\section{Methods}

\subsection{Subjects}

Eighty-seven home-based PD patients (54 males) were sequentially recruited from the Movement Disorders Clinic at the Perron Institute for Neurological and Translational Science (Perth, Australia) between 2008 and 2015. All patients were examined by a movement disorder neurologist prior to inclusion in the study for verification of the diagnosis in accordance with the UK Brain Bank criteria for IPD [21]. All PD patients were taking levodopa and 49 patients were also on a dopamine agonist (cabergoline, pramipexole, ropinirole or rotigotine). All PD medications were converted to levodopa equivalent daily doses (LEDD) [22]. Fourteen patients had implanted GPi deep brain stimulators (DBS). None of the patients had a history of liver disease or of a chronic inflammatory or iron storage disorder. For serum analysis, 36 age-matched healthy control samples were also collected. This study was approved by the Sir Charles Gairdner Hospital Human Research and Ethics Committee (Approval number 2006/073), and written informed consent was obtained from all participants, in accordance with the National Health and Medical Research Council guidelines.

\subsection{Clinical Assessments and Diagnosis of Impulse Control Disorders}

All clinical assessments were evaluated in the ' $\mathrm{ON}$ ' state. Motor symptoms were evaluated using the MDS-Unified Parkinson's Disease Rating Scale (MDS-UPDRS) Part III, and Hoehn and Yahr Scale [23]. In addition, each participant was evaluated by a clinical psychologist and completed a battery of neuropsychological assessments. The 'Barratt Impulsivity Scale 11' (BIS-11) was employed as a validated self-report questionnaire for screening impulsivity [24]. During the screening process, patients displaying any signs of an impulse control disorder were invited to seek further consultation with the clinical psychologist. Patients attending the subsequent consultation underwent a formal psychological evaluation, whereby a formal diagnosis of ICD was made based on the DSM-5 diagnostic criteria [25]. Venous blood samples were collected from patients and controls at the time of clinical assessment. For serum analysis of ferritin, transferrin, and iron, blood was collected from all PD patients and healthy controls.

\subsection{Determination of Sleep Problems}

Patient sleep disturbance was calculated by combining patient 'night-time sleep problems' (MDS-UPDRS item 1.7) and 'daytime sleepiness' (MDS-UPDRS 1.8). This combined score has previously been validated against other scales for measuring sleep disturbances, such as the 'Epworth Sleepiness Scale' and the 'PD Sleep Scale Version 2' (PDSS2) [26].

\subsection{Statistical Methods}

Data was analysed using IBM-SPSS (v. 24, IBM Corporation). A significant nominal $P$-value of $\leq 0.05$ was employed. One-way analysis of variance (ANOVA) tests with Tukey post-hoc analysis were used to compare the difference between defined impulsivity groups. In addition, the assessment for homogeneity of variance between groups was undertaken using Levene's test. In addition to the ANOVA, for the assessment of covariates such as age, an analysis of covariance (ANCOVA) was undertaken, and described where appropriate. For gender comparisons, and comparison between demographic variables, independent ttests or Mann-Whitney U were used.

\section{Results}

\subsection{Demographic and Clinical Correlates of Impulsivity in PD Patients}

The PD cohort enrolled in this study has previously been described [27], and was broadly ranging in age (68.9 \pm 31.8 $\mathrm{yrs})$, age of onset $(53.3 \pm 10.2 \mathrm{yrs})$, and disease duration $(10.4 \pm 6.8 \mathrm{yrs})$ and had no prior history of an ICD. In an effort to identify demographic and clinical indicators of patient impulsivity, the effects of gender, smoking history, PD medications and DBS surgery were examined and no 
significant associations were found with the total BIS-11 impulsivity scores (data not shown).

To determine if patient impulsivity was associated with other clinical markers in PD, BIS-11 scores were grouped into low $(<52)$, normal (52-71) and high levels $(>72)$ [24]. Given the previously reported link between recurrent falling and patient impulsivity in PD, this study first examined gender differences in disease severity, as determined by motor examination (MDS-UPDRS III). Patients selfreporting higher traits of impulsivity displayed a worsened disease severity, with significance only between groups in male PD patients $(p<.05)$. Given the established link between impulsivity and sleep disturbance, the authors subsequently investigated whether patients in this cohort exhibited a similar relationship. Sleep disturbance in patients, as measured by UPDRS 1.7 and 1.8 , was significantly associated with impulsivity groups $(p<.05)$. Sleep disturbance scores were significantly higher in the high impulsivity group $(p<.026)$, compared to patients in the normal-range group. Moreover, this association was observed in male, but not female patients (Figure 1B).

Table 1. Descriptive statistics of the Parkinson's Disease cohort $(n=87)$.

\begin{tabular}{ll}
\hline Clinical Characteristic/Assessment & Mean $( \pm$ SD) \\
\hline Age (Years) & $68.9(31.8)$ \\
Age of onset (Years) & $53.3(10.2)$ \\
BIS-11 & $61.8(10.0)$ \\
Disease duration (Years) & $10.4(6.8)$ \\
Daily LD equivalents (mg/day) & $671(389)$ \\
Hoehn Yahr (Stage 0-4) & $1.6(1.0)$ \\
MDS-UPDRS III Score & $12.8(14.2)$ \\
\hline
\end{tabular}

LD: Levodopa; UPDRS: Unified Parkinson's Disease Rating Scale

\subsection{Impulsivity Levels Relate to Serum Ferritin and Transferrin Saturation Levels}

Serum ferritin levels were elevated in the overall cohort of
PD patients $(n=87)$ when compared to healthy age-matched controls $(n=36)$, however this difference did not reach statistical significant. When the effect of gender was considered, elevated ferritin levels were observed in male PD patients and healthy controls, when compared to females $(p<.001)$, but there were no significant differences between PD patients and controls (Figure 2A). Serum ferritin levels showed a significant main effect between impulsivity groups $(p<.05)$, with a significant increase in the high impulsivity group when compared to the normal and low impulsivity groups $(p<.05)$. Male patients displayed a greater difference in serum ferritin between impulsivity groups $(p<.05)$, with an incremental rise in the three groups (Figure 2B). In contrast, female PD patients did not demonstrate the same significant relationship between impulsivity groups.

In the whole cohort of PD patients, serum iron and transferrin saturation were not significantly altered, when compared to healthy age-matched controls (Figure 2C and $2 \mathrm{E})$. When analysing the effect of gender, elevated iron levels were observed in male patients within the high impulsivity group (Figure 2D), but these results did not reach significance $(p=.054)$. Transferrin saturation (serum iron level $\times 100 /$ total iron-binding capacity) showed no difference in the low and normal range impulsivity groups. However, male patients in the high impulsivity group displayed significantly higher transferrin saturation levels than female patients $(p<.05)$ in the same group (Figure $2 \mathrm{~F}$ ).

To confirm these findings, ANCOVA analysis using age and LEDD as covariates were performed. In the male cohort, age and LEDD were not significant contributors to the observed variance in ferritin levels. Moreover, impulsivity (BIS-11) grouping was a significant variable $(p<.05)$, and pairwise comparisons of adjusted means revealed significance between high and normal impulsivity groups $(p=.010)$.
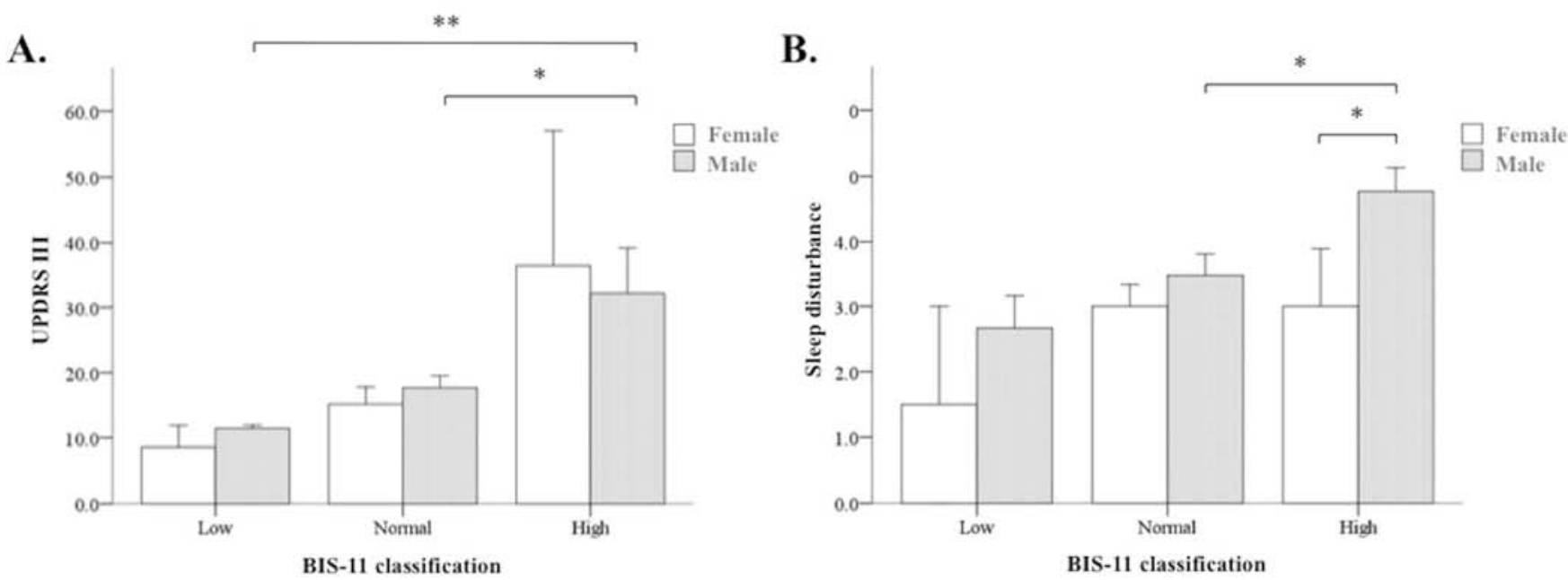

Figure 1. Clinical assessment indicators of impulsivity scores (BIS-11). When PD patients were grouped by impulsivity scores: (A) patients in the high impulsivity group had greater disease severity (MDS-UPDRS III), reaching statistical significance in male groups. (B) Gender-specific differences in sleep disturbance between impulsivity groups with an incremental increase in severity of sleep disturbance in males. Data shown are the mean \pm standard error; $*_{p}<.05: * *_{p}<.005$. 
A.

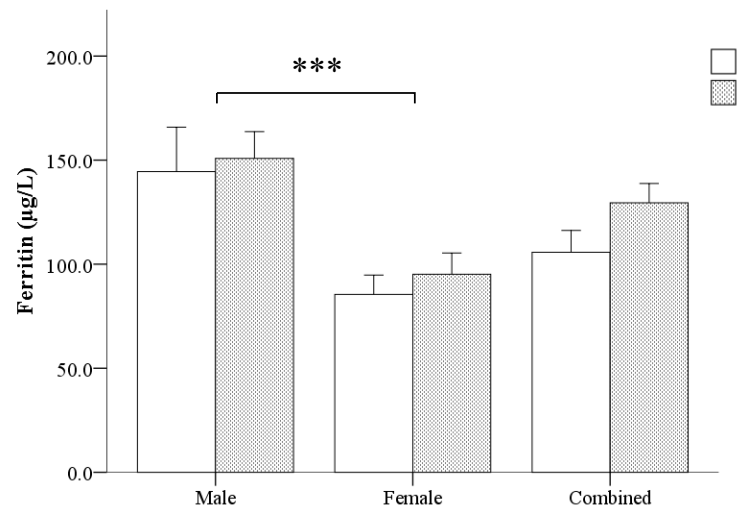

C.

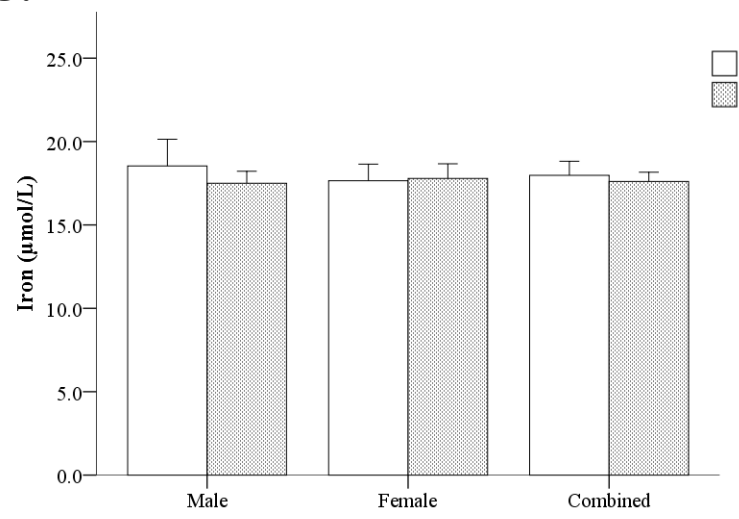

E.

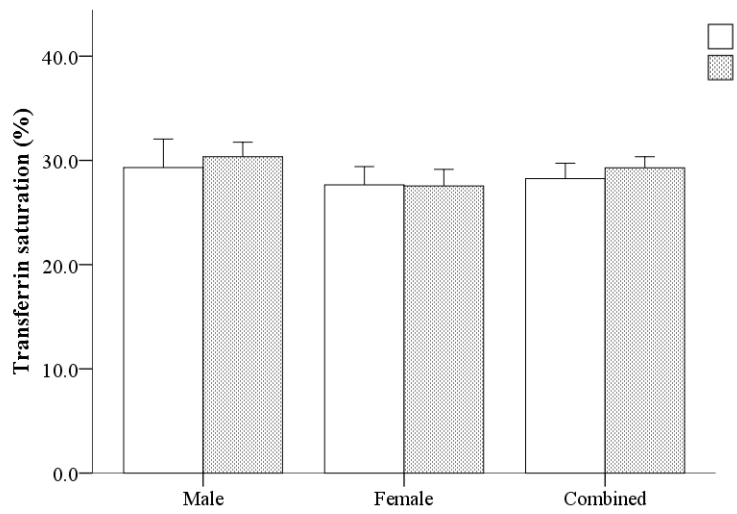

B.

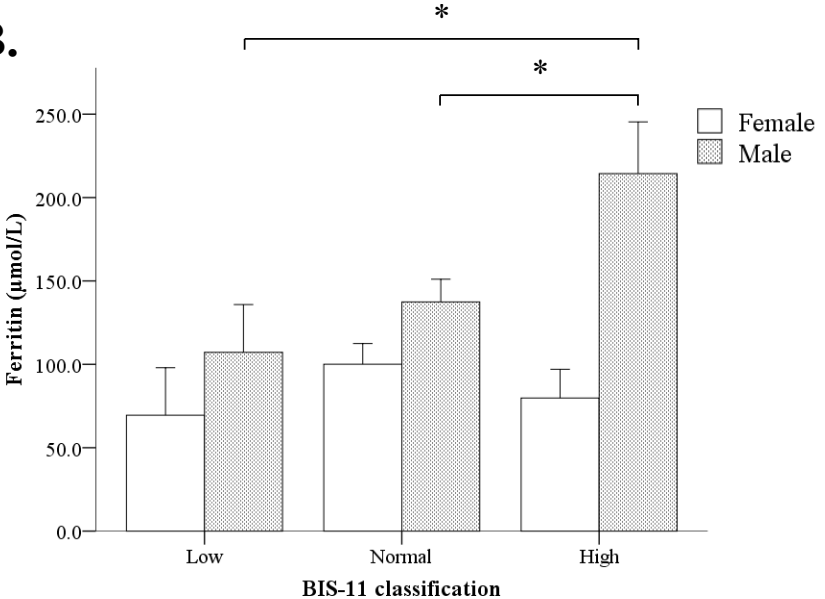

D.

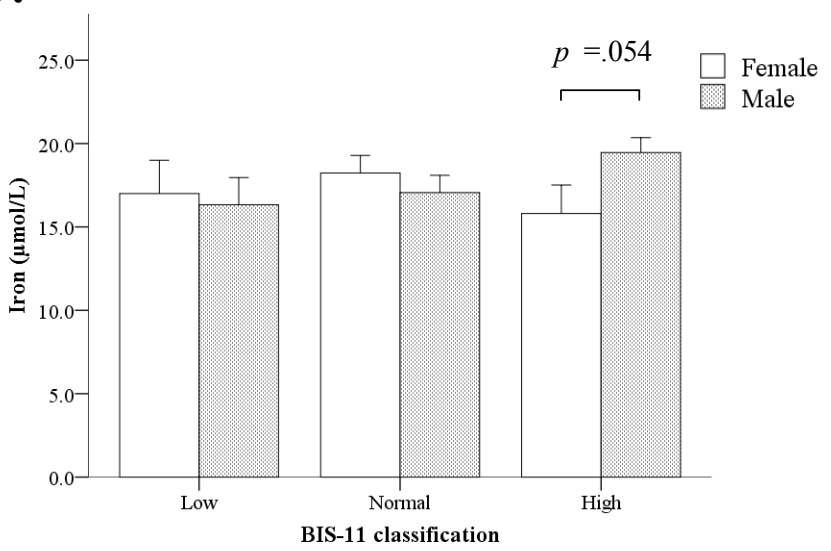

F.

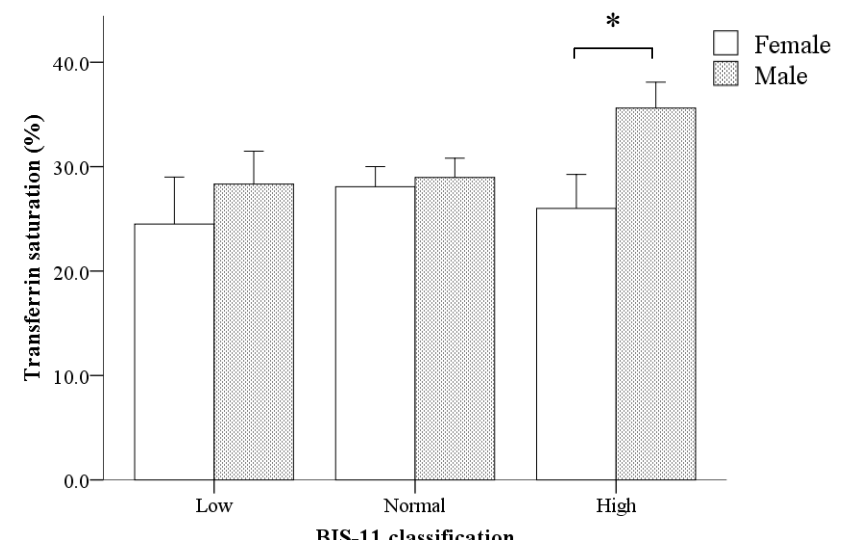

Figure 2. Serum ferritin, iron and transferrin saturation levels. (A) Ferritin levels were significantly higher in males than females both in the PD and control groups (B) Gender-specific differences in serum ferritin levels between impulsivity groups with an incremental rise in male PD patients but not females. (C) Serum iron levels were not significantly altered between control subjects compared with PD patients, or (D) between impulsivity groups. (E) Transferrin saturation percentage was not significantly altered between control subjects compared with PD patients. $(F)$ Within the high range impulsivity group, transferrin saturation percentage was elevated in males but not females. Data shown are the mean \pm standard error; ${ }^{*} p<.05, *^{* *} p<.005,{ }^{* * *} p<.001$.

\subsection{Subset of Patients Diagnosed with an ICD}

Following the preliminary psychological assessment and administration of the BIS-11, 11 patients (all males) with the highest impulsivity scores volunteered to undergo a more detailed evaluation by a clinical psychologist and were all found to meet the DSM-5 criteria for the diagnosis of an ICD. Their demographic and clinical features are detailed in Table 2 in comparison to the other 43 males in the PD cohort. 
Table 2. Baseline Clinical Characteristics of the male PD ( $n=43)$, and diagnosed ICD patient subset $(n=11)$.

\begin{tabular}{lll}
\hline Clinical Characteristic/Assessment & Male PD patients Mean ( \pm SD) & ICD subset Mean ( \pm SD) \\
\hline Age (Years) & $62.95(11.1)$ & $63.55(7.4)$ \\
Age of onset (Years) & $52.98(12.0)$ & $52.64(8.3)$ \\
Disease duration (Years) & $11.05(7.6)$ & $11.73(6.2)$ \\
Daily LD equivalents (mg/day) & $755.5(424.6)$ & $590.9(375.3)$ \\
Hoehn Yahr (Stage 0-4) & $1.67(0.9)$ & $1.73(1.1)$ \\
UPDRS III Score & $18.30(13.81)$ & $24.09(14.73)$ \\
\hline
\end{tabular}

LD: Levodopa; UPDRS: Unified Parkinson's Disease Rating Scale

A.

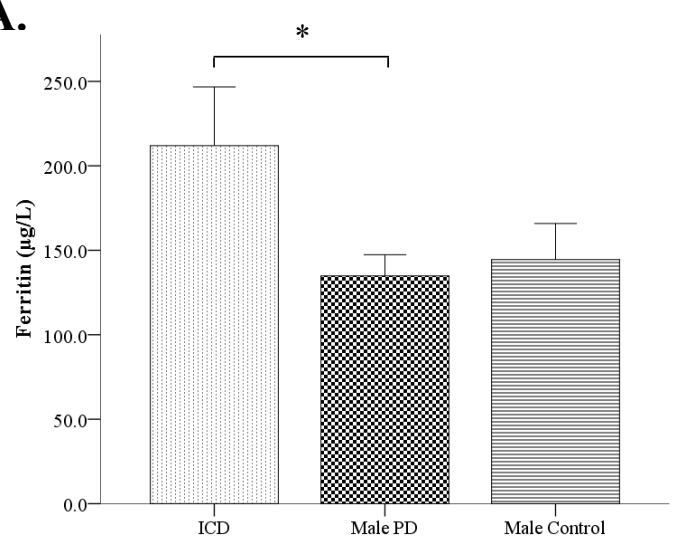

C.

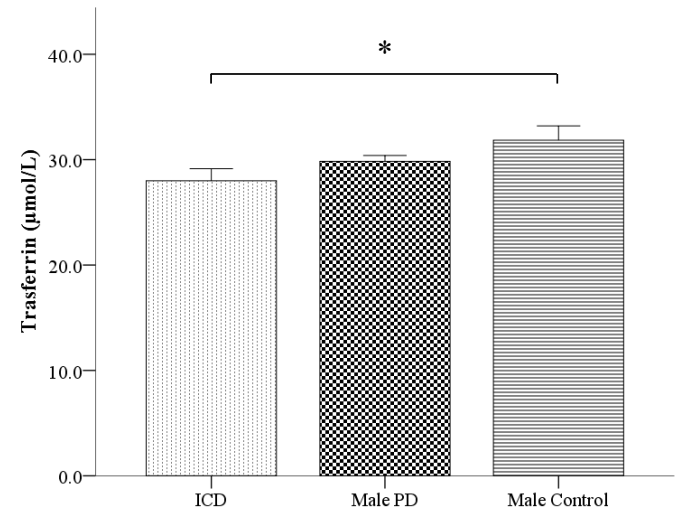

B.

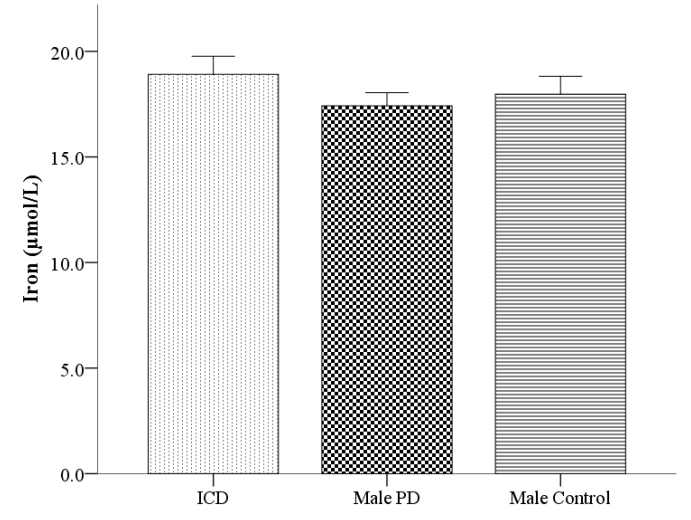

D.

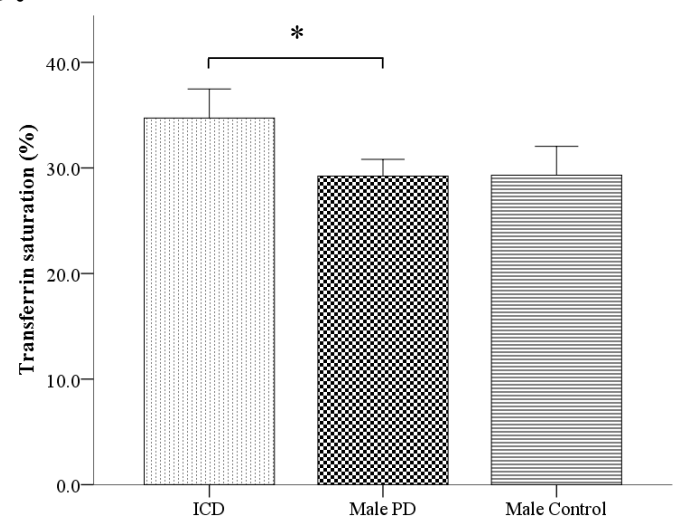

E.

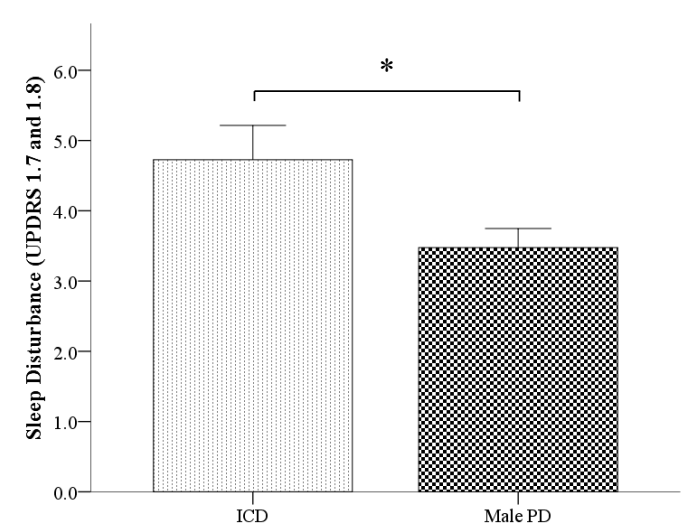

Figure 3. Comparative assessments of serum markers in ICD, male PD and male healthy controls. (A) Ferritin levels were significantly higher in ICD males compared to PD males, but not healthy controls (B) Serum iron levels were not significantly different between groups. (C) Transferrin levels were significantly reduced in the ICD group when compared with healthy control males. (D) Transferrin saturation was significantly elevated in the ICD cohort, when compared to males in the control and PD groups. (E) Patient sleep disturbance was significantly greater in males with an ICD compared with the male PD patients. Data shown are the mean \pm standard error; * $p<.05$. 


\subsection{Male ICD Patients show Higher Serum Ferritin Levels and Greater Sleep Disturbance}

To determine if findings relating to serum ferritin, iron, and transferrin saturation were also observed between groups, serum levels were compared between the subset of ICD patients, other male PD patients, and healthy male controls (Figure 3). Significantly higher ferritin levels and transferrin saturation, and significantly lower transferrin levels were observed in the ICD group when compared to the other male PD patients and healthy control males $(p<.05)$. Serum iron levels were significantly higher than in the other PD males, but not male healthy controls. Similarly, selfreported sleep disturbance (UPDRS 1.7 and 1.8) was greater in the ICD subset, when compared to the other PD male group $(p=.038)$.

\section{Discussion}

Findings from the present study demonstrated that when impulsivity scores were stratified into low, normal and high levels, male patients in the high impulsivity group had significantly greater motor impairment, as shown by higher UPDRS III scores, than males in the normal or low impulsivity groups. Interestingly, despite a similar trend with female patients, no significant differences in UPDRS III scores were observed between impulsivity groups. Similarly, the authors also observed a gender specific difference in the severity of sleep disturbance, with males in the high impulsivity group reporting greater disturbances then females. In addition, an incremental increase in sleep disturbance severity was observed in males across the three impulsivity groups. The basis for the association between sleep disturbance and impulsiveness is uncertain, specifically whether sleep disturbance increases impulsivity and the risk of developing an ICD, or is a consequence of it [1]. Rapid eye movement sleep associated behavioral disorder (RBD), which is common in PD patients, has also been associated with male gender, as well as longer disease duration, and medication doses [28]. While ICDs have recently been linked to RBD [29], polysomnography studies have failed to confirm an association between the two disorders, suggesting that they do not have a common underlying pathophysiology [30].

While the literature is divided on whether serum ferritin levels are elevated in PD, results from the present cohort indicated overall levels were higher in PD patients than controls, but these results did not reach statistical significance $(p=.056)$, while serum iron and transferrin saturation levels were comparable in the two groups. After stratifying for gender serum ferritin levels were found to be higher in males, both in the PD and control groups, as previously reported [31-33].

There are few studies linking ferritin and impulsivity, and the present study is the first to demonstrate an association between ferritin levels and high impulsivity in PD patients.
This was found to be gender-specific, in that it was present only in males, and there was a progressive increase in ferritin levels across the three impulsivity groups. Males in the high impulsivity group, who also had the greatest degree of sleep disturbance, had the highest ferritin levels $(p<.05)$ as well as increased transferrin saturation $(p<.05)$ and raised serum iron levels $(p=.054)$, in keeping with a state of relative iron overload.

On the basis of the initial BIS-11 screening assessment we identified a subset of male patients with high impulsivity scores who fulfilled the diagnostic criteria for an ICD. This subset consisted of 11 male patients who were diagnosed by a clinical psychologist and appeared to present with a somewhat greater disease severity (UPDRS III score), when compared to the remaining male PD patient cohort. Unlike previous studies, the ICD subset were not of early disease onset or longer disease duration [3], with findings showing age, disease duration and age of disease not being significant variables of ICD prevalence in PD. Despite being of similar age and disease duration, the ICD subset also displayed significantly greater ferritin levels and transferrin saturation when compared to the other male PD patients $(p<.05)$, and elevated transferrin saturation compared to healthy controls. Moreover, consistent with the high impulsivity group, as determined by BIS-11 scores, the ICD subset reported significantly greater sleep disturbance than male PD patients $(p<.05)$. These results are somewhat surprising, as low brain iron levels have been associated with increased sleep disturbances and sleep fragmentation [34], a feature documented in RLS [35]. However, it is known that patients suffering from iron overload syndromes can also experience similar sleep-related difficulties [36].

\section{Limitations}

Patient medication was optimised, and patient selection was made on the basis of independence and mobility in that those unable to walk unassisted were excluded, as demonstrated by a comparatively low mean cohort Hoehn and Yahr score. A number of limitations need to be acknowledged. Firstly, while validated against standard measures of sleep quality [26], interpretations of sleep disturbance using self-reported questions from the MDSUPDRS should be considered with caution. Secondly, following initial evaluation, not all patients with high impulsivity scores volunteered to undergo a more detailed follow-up consultation with a clinical psychologist and the incidence of ICD may have been underestimated. As such, a 'non-ICD' patient subset could not be accurately determined, and the manuscript acknowledges this limitation by comparing the ICD group with the other male patients in the cohort. Thirdly, although none of the PD patient cohort were known to have any other condition that might be associated with an elevated serum ferritin level, laboratory investigations to exclude secondary causes of raised ferritin levels were not carried out. Lastly, in view of the multiple 
factors which could contribute to a high impulsivity score in a chronic disease such as $\mathrm{PD}$, including premorbid personality traits and subsequent effects of the disease itself and medications, and in view of the cross-sectional nature of this study, caution needs to be exercised in equating high impulsivity levels as demonstrated by the BIS-11 screening tool with the development of an ICD. Nevertheless, it is noteworthy that all of the male patients with the highest impulsivity scores were in fact found to fulfill the criteria for an ICD on further evaluation.

\section{Conclusions}

In conclusion, using the BIS-11 screening tool, this study demonstrated that male PD patients with the highest impulsivity levels had significantly higher serum ferritin levels and transferrin saturation, and also experienced the most severe sleep disturbance. These associations were also present in a subset of males who met the DSM-5 criteria for an ICD. Although the findings do not necessarily imply a causal relationship between high ferritin levels and high levels of impulsivity and ICDs they suggest that there may be a link between altered iron homeostasis and the development of these disorders in males with PD, which warrants further investigation. These findings also indicate the value of the BIS-11 scale as a screening tool to identify patients who may have an ICD who require further evaluation and treatment.

\section{Acknowledgements}

The authors declare no conflicts of interest. The study was funded by Grant / Research support from the federal Cooperative Research Centre for Mental Health, the Perron Institute for Neurological and Translational Science, and the University of Notre Dame Australia.

\section{References}

[1] Djamshidian, A., W. Poewe, and B. Högl, Impact of Impulse Control Disorders on Sleep-Wake Regulation in Parkinson's Disease. Parkinson's Disease, 2015. 2015: p. 970862.

[2] Garcia-Ruiz, P. J., et al., Impulse control disorder in patients with Parkinson's disease under dopamine agonist therapy: a multicentre study. Journal of Neurology, Neurosurgery \& Psychiatry, 2014. 85(8): p. 840-844.

[3] Abosch, A., et al., Impulsive Behavior and Associated Clinical Variables in Parkinson's Disease. Psychosomatics, 2011. 52(1): p. 41-47.

[4] Smulders, K., et al., Trait Impulsivity Is Associated with the Risk of Falls in Parkinson's Disease. PLoS ONE, 2014. 9(3): p. e91190.

[5] Killgore, W. D. S., et al., Sleep deprivation reduces perceived emotional intelligence and constructive thinking skills. Sleep Medicine, 2008. 9(5): p. 517-526.

[6] Kumar, S., M. Bhatia, and M. Behari, Sleep disorders in Parkinson's disease. Movement Disorders, 2002. 17(4): p.
$775-781$.

[7] Happe, S., et al., Sleep disorders and depression in patients with Parkinson's disease. Acta Neurologica Scandinavica, 2001. 104(5): p. 275-280.

[8] Chaudhuri, K. R. and K. Logishetty, Dopamine receptor agonists and sleep disturbances in Parkinson's disease. Parkinsonism \& Related Disorders, 2009. 15: p. S101-S104.

[9] Scullin, M. K., et al., Sleep and Impulsivity in Parkinson's Disease. Parkinsonism \& related disorders, 2013. 19(11): p. 10.1016/j.parkreldis.2013.06.018.

[10] Paavonen, E. J., et al., Short Sleep Duration and Behavioral Symptoms of Attention-Deficit/Hyperactivity Disorder in Healthy 7- to 8-Year-Old Children. Pediatrics, 2009. 123(5): p. e857.

[11] Pourcher, E., S. Rémillard, and H. Cohen, Compulsive habits in restless legs syndrome patients under dopaminergic treatment. Journal of the Neurological Sciences, 2010. 290(1): p. $52-56$.

[12] Schulte, E. C., et al., Iron in Restless Legs Syndrome. Movement Disorders Clinical Practice, 2014. 1(3): p. 161172 .

[13] Cortese, S., et al., Sleep disturbances and serum ferritin levels in children with attention-deficit/hyperactivity disorder. European Child \& Adolescent Psychiatry, 2009. 18(7): p. 393399.

[14] Oner, P., et al., Ferritin and Hyperactivity Ratings in Attention Deficit Hyperactivity Disorder. Pediatrics international: official journal of the Japan Pediatric Society, 2012. 54(5): p. 688-692.

[15] Mochizuki, H. and T. Yasuda, Iron accumulation in Parkinson's disease. Journal of Neural Transmission, 2012. 119(12): p. 1511-1514.

[16] Friedman, A., et al., Ferritin as an important player in neurodegeneration. Parkinsonism \& Related Disorders, 2011. 17(6): p. 423-430.

[17] Bartzokis, G., et al., Brain Ferritin Iron as a Risk Factor for Age at Onset in Neurodegenerative Diseases. Annals of the New York Academy of Sciences, 2004. 1012(1): p. 224-236.

[18] Farhoudi, M., et al., Serum iron and ferritin level in idiopathic Parkinson. Pak J Biol Sci, 2012. 15(22): p. 1094-7.

[19] Medeiros, M. S., et al., Iron and Oxidative Stress in Parkinson's Disease: An Observational Study of Injury Biomarkers. PLoS ONE, 2016. 11(1): p. e0146129.

[20] Ikeda, K., et al., Serological Profiles of Urate, Paraoxonase-1, Ferritin and Lipid in Parkinson's Disease: Changes Linked to Disease Progression. Neurodegenerative Diseases, 2011. 8(4): p. 252-258.

[21] Hughes, A. J., et al., Accuracy of clinical diagnosis of idiopathic Parkinson's disease: a clinico-pathological study of 100 cases. J Neurol Neurosurg Psychiatry, 1992. 55(3): p. 181-4.

[22] Tomlinson, C. L., et al., Systematic review of levodopa dose equivalency reporting in Parkinson's disease. Movement Disorders, 2010. 25(15): p. 2649-2653. 
[23] Goetz, C. G., et al., Movement Disorder Society-sponsored revision of the Unified Parkinson's Disease Rating Scale (MDS-UPDRS): Process, format, and clinimetric testing plan. Movement Disorders, 2007. 22(1): p. 41-47.

[24] Patton, J. H., M. S. Stanford, and E. S. Barratt, Factor structure of the Barratt impulsiveness scale. J Clin Psychol, 1995. 51(6): p. 768-74.

[25] Association, A. P., Diagnostic and statistical manual of mental disorders: DSM-5, ed. A. American Psychiatric and D. S. M. T. F. American Psychiatric Association. 2013, Arlington, VA: American Psychiatric Association.

[26] Horvath, K., et al., Is the MDS-UPDRS a Good Screening Tool for Detecting Sleep Problems and Daytime Sleepiness in Parkinson's Disease? Parkinson's Disease, 2014. 2014: p. 8.

[27] Evans, T., et al., Extended "Timed Up and Go" assessment as a clinical indicator of cognitive state in Parkinson's disease. Journal of the Neurological Sciences, 2017. 375: p. 86-91.

[28] Sixel-Doring, F., et al., Associated factors for REM sleep behavior disorder in Parkinson disease. Neurology, 2011. 77(11): p. 1048-1054.

[29] Bellosta Diago, E., et al., Relación entre el trastorno de conducta del sueño REM y el trastorno de control de impulsos en pacientes con enfermedad de Parkinson. Neurología, 2016.
[30] Bayard, S., et al., Impulse control disorder and rapid eye movement sleep behavior disorder in Parkinson's disease. Parkinsonism \& Related Disorders, 2014. 20(12): p. 1411-1414.

[31] Jehn, M., J. M. Clark, and E. Guallar, Serum Ferritin and Risk of the Metabolic Syndrome in U.S. Adults. Diabetes Care, 2004. 27: p. 2422-2428.

[32] Zacharski, L. R., et al., Association of age, sex, and race with body iron stores in adults: Analysis of NHANES III data. American Heart Journal, 2000. 140(1): p. 98-104.

[33] Dekker, L. H., et al., Sex Differences in the Association Between Serum Ferritin and Fasting Glucose in Type 2 Diabetes Among South Asian Surinamese, African Surinamese, and Ethnic Dutch. Diabetes Care, 2013. 36(4): p. 965.

[34] Youssef, J., et al., Relationship of Serum Ferritin Levels to Sleep Fragmentation and Periodic Limb Movements of Sleep on Polysomnography in Autism Spectrum Disorders. Pediatric Neurology, 2013. 49(4): p. 274-278.

[35] Daubian-Nosé, P., M. K. Frank, and A. M. Esteves, Sleep disorders: A review of the interface between restless legs syndrome and iron metabolism. Sleep Science, 2014. 7(4): p. 234-237.

[36] Moirand, R., et al., A new syndrome of liver iron overload with normal transferrin saturation. The Lancet, 1997. 349(9045): p. 95-97. 